\title{
A reflective process led by a family physician to develop a renal-protection surveillance tool for HIV patients newly started on dolutegravir
}

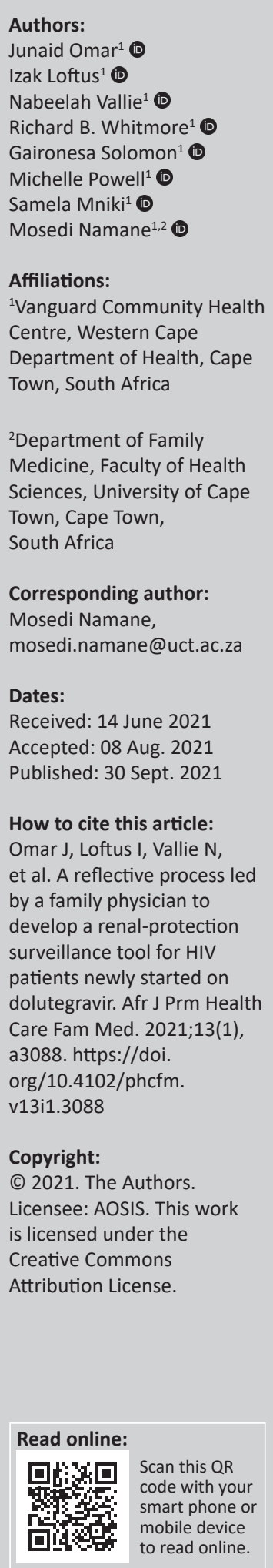

A group of Vanguard Community Health Centre doctors embarked on a Health System's Improvement (HSI) project with the aim of reducing harm to renal function in patients who were either commenced on or switched to a dolutegravir (DTG)-based antiretroviral therapy (ART) regimen since 2019, when the usual monitoring and evaluation of ART-regimen switches were disrupted by the coronavirus disease 2019 (COVID-19) pandemic. This intended harm-reduction exercise, involving a reflective process that was facilitated by the family physician, led to the development of a Vanguard Renal Protection Surveillance tool, which is now used at Vanguard to detect and prevent renal decline.

Keywords: HIV; renal protection; health system improvement; South Africa; Dolutegravir.

\section{Introduction}

Vanguard Community Health Centre (VCHC) is a primary care facility in the Western Cape (WC), South Africa (SA). During the first wave of the coronavirus disease 2019 (COVID-19) in 2020, the facility began switching HIV-positive people from an efavirenz-based first line antiretroviral regimen (EFV-BR) to a dolutegravir-based regimen (DTG-BR). Because the COVID-19 demanded additional attention from health care workers (HCWs), the switch lacked the usual rigour in monitoring adverse events that may come with the introduction of new medicines. ${ }^{1}$ Informal and unofficial local feedback from colleagues alleged that there could be serious decline in renal function associated with this DTG-BR. This decline was said not to be related to the known and expected increase in serum creatinine of less than $15 \%$ from dolutegravir $(\mathrm{DTG}){ }^{2}$ nephrotoxicity from tenofovir (TDF) ${ }^{3}$ or HIV-associated nephropathy. ${ }^{4}$ The concern of possible nephrotoxicity, associated with this new medication, prompted the authors at VCHC to develop a special surveillance system for detecting early renal impairment, so that if necessary, they could implement earlier remedial actions. The project was led and coordinated by the facility's family physician (FP), who had experiences of several other WC Health System's Improvement (HSI) programmes. .,6,7 $^{-}$

\section{Development of the surveillance tool for renal function}

The aim of the initiative was to develop a surveillance tool that could assist clinicians to prevent and/or detect early renal decline in patients who had been commenced on a DTG-based regimen. The tool was developed through rounds of piloting, reflection and discussions amongst the authors and other practitioners at VCHC (in the main, the nurses). This was also seen as an opportunity to train staff on adverse drug reporting (ADR).

The first task was the drafting of the Vanguard Renal protection Surveillance Tool (VanReST). The tool included potential risk factors for renal disease that had been identified from the SA Primary Health care and Adult Standard Treatment Guidelines. ${ }^{8,9}$ The VanReST (Figure 1) was used to audit folders of HIV-positive people (ranging from children/adolescent $\geq 35 \mathrm{~kg}$ and $\geq 10$ years to adults), who had been commenced on or switched to a fixed regimen of tenofovir/lamivudine/ dolutegravir (TLD) since 2019. The audit intended to review 60 systematic randomly selected folders.

Three medical officer and community-service medical officer pairs were allocated 20 folders to review using VanReST. Protected time was set aside to allow each duo to audit five folders per session, and then reflect on their experience and document their feedback. These reflections were 


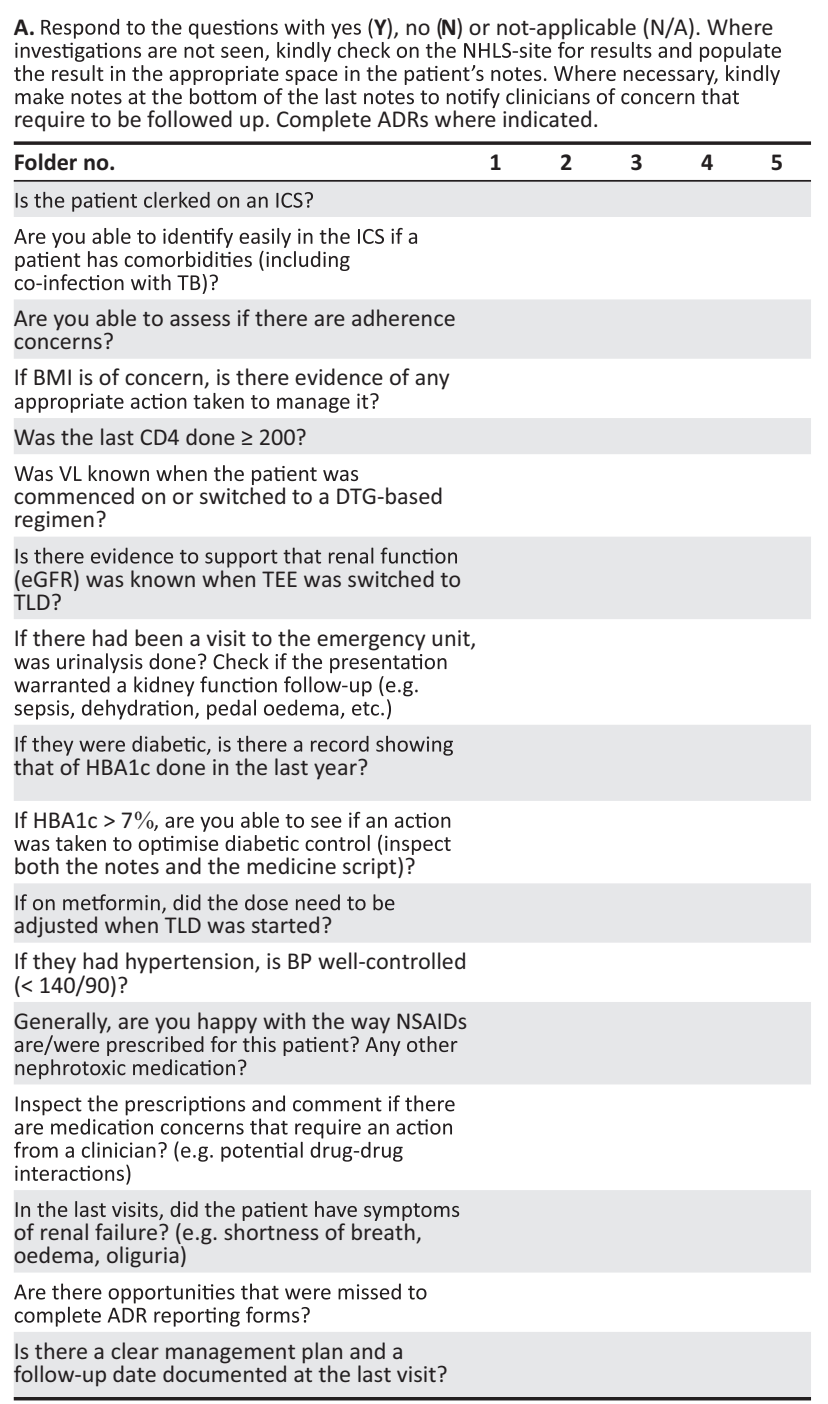

NHLS, National Health Laboratory Service; ICS, Integrated Care stationery for primary care in the Western Cape; TB, tuberculosis; BMI, body mass index; VL, viral load; eGFR, estimated Glomerular Filtration Rate; TEE, fixed dose oral medication that includes tenofovir disoproxi fumarate, emtricitabine and efavirenz; TLD, fixed dose oral medication that includes tenofovir disoproxil fumarate, lamivudine and DTG; HBA1c, haemoglobin A1c test that measures the amount of blood sugar attached to haemoglobin; BP, blood pressure; ADR, adverse drug reaction.

FIGURE 1: Vanguard renal protection surveillance tool for patients on antiretroviral therapy.

shared with the whole team and were used to refine the tool over several rounds. Once all the folders had been reviewed, the documented feedback was handed in and thematically analysed by the FP and a medical officer. The findings were presented to the HIV unit staff, most of whom had special training in antiretroviral therapy (ART) management and they also gave feedback on the project.

Only 22 folders had a complete set of medical notes. The researchers however, felt that the surveillance tool (Figure 1) was fully developed and reviewing more folders would not refine it further. Table 1 summarises the key themes from the feedback: support for use of tool; poor record keeping as the main barrier to monitor renal function and lack of common understanding of the revised ART protocols with DTG.
TABLE 1: Reflections on the project, recommendations, and improvement plans.

\begin{tabular}{|c|c|c|}
\hline Theme & Quote & $\begin{array}{l}\text { Recommendations and } \\
\text { improvement plans }\end{array}$ \\
\hline $\begin{array}{l}\text { VanReST was found to } \\
\text { be useful in promoting } \\
\text { renal health and its } \\
\text { continued use was } \\
\text { supported }\end{array}$ & $\begin{array}{l}\text { 'From limited data, it seems } \\
\text { that there is no increase (in } \\
\text { month 4, creatinine compared } \\
\text { to baseline level).' (D2, } \\
\text { CS3, M) } \\
\text { 'It helped to create awareness } \\
\text { around possible renal } \\
\text { impairment in patients on } \\
\text { TLD.' (D3, MO4, F) } \\
\text { 'We need to use this } \\
\text { information to implement } \\
\text { changes that can address } \\
\text { these gaps.' (D1, MO2, F) } \\
\text { 'Enables us to monitor all the } \\
\text { patients on TLD and report } \\
\text { any ADRs.' (D3, MO4, F) }\end{array}$ & $\begin{array}{l}\text { VanResT to be adopted at } \\
\text { Vanguard and be made a } \\
\text { desk-top essential in all } \\
\text { consulting rooms. } \\
\text { ADR training implemented } \\
\text { during the period of study } \\
\text { The FP to champion } \\
\text { surveillance }\end{array}$ \\
\hline $\begin{array}{l}\text { A cascade of challenges } \\
\text { experienced in locating } \\
\text { and/or obtaining } \\
\text { complete medical } \\
\text { records disrupted } \\
\text { continuity of care and } \\
\text { made it difficult to do } \\
\text { a comprehensive } \\
\text { renal-risk surveillance. }\end{array}$ & $\begin{array}{l}\text { 'Procuring the folders has } \\
\text { been a challenge.' (D1, } \\
\text { MO1, M) } \\
\text { 'Outside folders were difficult } \\
\text { to find.' (D2, CS2, F) } \\
\text { 'Why are the inners } \\
\text { (ART-clinic visit notes) and } \\
\text { outers (cover of folder \& rest } \\
\text { of notes) filed separately?.' } \\
\text { (V, PN1, F) } \\
\text { 'We see patients on what is } \\
\text { referred to as duplicates, you } \\
\text { cannot call a new folder a } \\
\text { duplicate ... there is nothing } \\
\text { there.' (V, PN2, F) }\end{array}$ & $\begin{array}{l}\text { Unit manager and clinicians } \\
\text { to campaign for a reliable } \\
\text { storage and retrieval of } \\
\text { folders. }\end{array}$ \\
\hline $\begin{array}{l}\text { Comments that } \\
\text { indicated that there } \\
\text { was no common } \\
\text { understanding of the } \\
\text { latest ART protocols } \\
\text { amongst clinicians }\end{array}$ & $\begin{array}{l}\text { 'Also, from my time in ARV } \\
\text { clinic, it seems like they aren't } \\
\text { strictly doing renal functions } \\
\text { after starting DTG like we } \\
\text { expected.' (D2, MO3, F) } \\
\text { 'After switching from TEE to } \\
\text { TLD, the VL, creatinine, eGFR } \\
\text { are done routinely, that is, } \\
\text { after } 12 \text { months and annully } \\
\text { as per guidelines. No extra } \\
\text { bloods are taken.' (D3, MO4, F) }\end{array}$ & $\begin{array}{l}\text { SM (our infectious diseases } \\
\text { champion) to present } \\
\text { updates of the latest ART } \\
\text { protocols and ensure every } \\
\text { clinician has a correct copy. }\end{array}$ \\
\hline
\end{tabular}

D1, reflections from document $1 ; D 2$, reflections from document 2; D3, reflections from document $3 ; \mathrm{V}$, Verbal comments from stakeholders in a feedback session; CS, community service doctor; MO, medical officer; PN, professional nurse; $m$, male; $F$, female; VanReST Vanguard Renal Protection Surveillance Tool; TEE, fixed dose oral medication that includes tenofovir disoproxil fumarate, emtricitabine and efavirenz; TLD, fixed dose oral medication that includes tenofovir disoproxil fumarate, lamivudine and dolutegravir; DTG, dolutegravir $A D R$, adverse drug reaction; ART, antiretroviral therapy; VL, viral load; eGFR, estimated glomerular filtration rate.

\section{The family physician's reflections on the journey taken to address an 'unavoidable' serious concern}

It was necessary to lead by being 'present' and not allow the overwhelming COVID-19 pandemic activities to derail the process. The methodology unfolded 'as we went along' whilst I held focus for the team. The main challenge encountered at the initial stages was to convince stakeholders that the project was not meant to generate nor analyse quantitative data, but rather to 'clean up' the whole system related to renal health.

A bonus of this project has been two-fold: firstly, it gave our young doctors opportunities to experience distributive leadership, to engage in reflective exercises and to advocate for a safe, accessible and continuous healthcare service for HIV-positive people. Secondly, since starting to use the tool, the nurses seem to appreciate the importance of evaluating one's own practice. They were increasingly reporting on blind spots they identified and gaps that they were closing.

This short report demonstrates how a FP can lead the team in terms of clinical governance to improve quality of care and 
patient safety. In this example, audit and feedback not only improved care, but also developed a useful tool that could be used going forward. At the same time, the FP capacitated the team of medical officers and nurses to develop skills in quality improvement, monitoring and evaluation. The exercise highlighted the importance of informational continuity that is essential for high quality primary care and the need to implement new clinical guidelines.

\section{Conclusion}

A renal protection surveillance system was developed and implemented at VCHC out of a concern for possibly missing cases of nephrotoxicity during the disruptions of health services during the intersection of the HIV and coronavirus pandemics. The VCHC staff believe that the potential of the surveillance tool will be enhanced by an improved medical record management system. The report illustrates the contribution of FPs to clinical governance and leadership within a primary healthcare team.

\section{Acknowledgements}

We acknowledge Vanguard nurses and medical interns who contributed to the discussions.

\section{Competing interests}

The authors declare that they have no financial or personal relationships that may have inappropriately influenced them in writing this article.

\section{Authors' contributions}

M.N. supervised and coordinated the whole project and the writing and editing of the article. J.O. assisted with the supervision of the study and data analysis. I.J. proposed the research and managed timelines for the study. All authors contributed equally to conceptualising the study, in protocol writing, in the research and in analysis of data.

\section{Ethical considerations}

This narrative project was stimulated by a call by the African Journal of Primary Health Care $\mathcal{E}$ Family Medicine (PHCFM) to submit a short report on how they contribute to African health systems. M.N., as the head of clinical governance at the Community Health centre, approved that this project of developing a tool to prevent and to survey for early nephrotoxicity amongst people living with HIV be shared in PHCFM as an example of health systems improvement led by a family physician in a large Community Health centre serving an average of 30000 patients per month.

\section{Funding information}

The authors received no financial support for the research, authorship and/or publication of this article.

\section{Data availability}

The sources for the criteria to survey renal health are South African National Standard treatment guidelines that are referenced in the article. The typed reflective statements of the researchers are available electronically.

\section{Disclaimer}

The views and opinions expressed in this article are those of the authors and do not necessarily reflect the official policy or position of University of Cape Town or Western Cape Department of Health.

\section{References}

1. WHO. Dolutegravir (DTG) and the fixed dose combination of tenofovir/lamivudine/ dolutegravir (TLD). Briefing Note, April 2018. Geneva: WHO; 2018.

2. Maggi $P$, Montinaro V, Mussini $C$, Biagio A Di, Bellagamba R. Novel antiretroviral drugs and renal function monitoring of HIV patients. AIDS Rev. 2014;16(3):144-151.

3. Mtisi TJ, Ndhlovu CE, Maponga CC, Morse GD. Tenofovir - Associated kidney disease in Africans: A systematic review. AIDS Res Ther. 2019;16:12. https://doi. org/10.1186/s12981-019-0227-1

4. Brook MG, Miller RF, Clements P. HIV associated nephropathy: A treatable condition. Sex Transm Infect. 2001;77(2):97-100. https://doi.org/10.1136/ sti.77.2.97

5. Solomon $G$, Allie A, Fakier R, et al. Family medicine internship support during the COVID-19 pandemic in Cape Town, South Africa - A narrative report. Afr J Prim Health Care Fam Med. 2020;12(1):a2661. https://doi.org/10.4102/phcfm. v12i1.2661

6. Coetzee R, Johnson $\mathrm{Y}$, Niekerk J Van, Namane M. Amitriptyline prescribing in public sector healthcare facilities in the Western Cape, South Africa. PLoS One. 2020;15(4):e0231675. https://doi.org/10.1371/journal.pone.0231675

7. Govender I, Ehrlich R, Van Vuuren U, et al. Clinical audit of diabetes management can improve the quality of care in a resource-limited primary care setting. Int J Health Care Qual Assur. 2012;24(6):612-618. https://doi.org/10.1093/intqhc/ mzs063

8. Primary Healthcare Level. Standard treatment guidelines and essential medicines list for South Africa, 2020 [homepage on the Internet]. 2020 [cited
2021 Mar 05]. Available from: http://www.kznhealth.gov.za/pharmacy/PHC2021 Mar 05
STG-2020.pdf

9. Hospital Level (Adults) Standard Treatment Guidelines and Essential Medicines List 2nd Edition - 2019 [homepage on the Internet]. 2019 [cited 2021 Mar 05] Available from: https://www.knowledgehub.org.za/elibrary/hospital-level-adultsAvailable from: https://www.knowledgehub.org.za/elibrary/hosp
standard-treatment-guidelines-and-essential-medicines-list-2nd 